OPEN ACCESS

Edited by:

Xiang Xie,

First Affiliated Hospital of Xinjiang Medical University, China

Reviewed by: Jose Francisco Huizar Hunter Holmes McGuire VA Medical Center, United States

Mona Mostafa Rayan, Ain Shams University, Egypt Xuejuan Jin,

Fudan University, China

${ }^{*}$ Correspondence: Ping-Yen Liu larry@mail.ncku.edu.tw

Specialty section

This article was submitted to General Cardiovascular Medicine, a section of the journal Frontiers in Cardiovascular Medicine

Received: 19 October 2021 Accepted: 31 December 2021

Published: 03 February 2022

Citation:

Lee $P$-T, Huang T-C, Huang M-H, Hsu L-W, SU P-F, Liu Y-W, Hung M-H and Liu P-Y (2022) The Burden of Ventricular Premature Complex Is Associated With Cardiovascular Mortality.

Front. Cardiovasc. Med. 8:797976.

doi: 10.3389/fcrm.2021.797976

\section{The Burden of Ventricular Premature Complex Is Associated With Cardiovascular Mortality}

\author{
Po-Tseng Lee 1,2, Ting-Chun Huang 1,2, Mu-Hsiang Huang ${ }^{2}$, Ling-Wei Hsu ${ }^{1}$, Pei-Fang Su ${ }^{3}$, \\ Yen-Wen Liu ${ }^{1,2}$, Meng-Hsuan Hung ${ }^{1}$ and Ping-Yen Liu ${ }^{1,2 *}$ \\ ${ }^{1}$ Institute of Clinical Medicine, College of Medicine, National Cheng Kung University, Tainan, Taiwan, ${ }^{2}$ Division of Cardiology, \\ Department of Internal Medicine, National Cheng Kung University Hospital, College of Medicine, National Cheng Kung \\ University, Tainan, Taiwan, ${ }^{3}$ Department of Statistics, College of Management, National Cheng Kung University, Tainan, \\ Taiwan
}

Background: Ventricular premature complex (VPC) is one of the most common ventricular arrhythmias. The presence of VPC is associated with an increased risk of heart failure (HF).

Method: We designed a single-center, retrospective, and large population-based cohort to clarify the role of VPC burden in long-term prognosis in Taiwan. We analyzed the database from the National Cheng Kung University Hospital-Electronic Medical Record (NCKUH-EMR) and NCKUH-Holter (NCKUH-Holter). A total of 19,527 patients who underwent 24-h Holter ECG monitoring due to palpitation, syncope, and clinical suspicion of arrhythmias were enrolled in this study.

Results: The clinical outcome of interests involved 5.65\% noncardiovascular death and 1.53\% cardiovascular-specific deaths between 2011 and 2018. Multivariate Cox regression analysis, Fine and Gray's competing risk model, and propensity score matching demonstrated that both moderate (1,000-10,000/day) and high (>10,000/day) VPC burdens contributed to cardiovascular death in comparison with a low VPC burden $(<1,000 /$ day $)$.

Conclusion: A higher VPC burden via Holter ECG is an independent risk factor of cardiovascular mortality.

Keywords: ventricular premature complex (VPC), 24-h Holter electrocardiogram, cardiovascular mortality, cox regression analysis, Fine and Gray's model

\section{INTRODUCTION}

Ventricular premature complex (VPC) is one of the most common ventricular arrhythmias and is strongly associated with heart failure (HF), all-cause hospitalization, and cardiovascular hospitalization (1-3). A high VPC burden (>10,000/day) can reduce systolic blood pressure and is reportedly detrimental to the left ventricular systolic function $(2,4,5)$. The accumulated data demonstrate that the elimination of VPC by either catheter ablation or antiarrhythmic drugs (AADs) not only relieves symptoms but also improves the left ventricular systolic function in patients with preexisting left ventricular systolic dysfunction or VPC-induced cardiomyopathy (610). Of interest, cardiac mortality, transplantation, and hospitalization are reduced in patients with frequent VPC after receiving a successful catheter ablation (11). 
Regarding the overall effect of VPC burden on the cardiovascular system, several limitations exist in the current research literature. First, the size of previous cohorts using 24h Holter monitoring to evaluate daily VPC burden is limited that the largest population size reported to date is 5,778 patients in a single cohort (12). Although the other all-comer studies and meta-analysis contained a large population size, the lack of daily VPC counts creates difficulty in evaluating the relationship between daily burden and prognosis (13). Second, several significant clinical comorbidities could be confounding factors for all-cause death and cardiovascular death. These factors include age, coexisting diseases like diabetes mellitus (DM), chronic kidney disease (CKD), and stroke, the presence of ventricular tachycardia, and HF. Third, although the presence of exercise-induced VPC was reported to be associated with cardiovascular death, the effect of VPC burden on cardiovascular death has not been directly reported until a recent subgroup analysis from the CHF-STAT study demonstrated that amiodarone could suppress PVC burden and improve survival $(10,13,14)$. To consolidate the role of VPC burden on patient prognosis, particularly cardiovascular death, we designed a single-center, retrospective study with a large populationbased cohort.

\section{ORIGINAL RESEARCH}

\section{Materials and Methods Data Source and Study Population}

This retrospective, observational study utilized the National Cheng Kung University Hospital-Electronic Medical Record (NCKUH-EMR) database and the NCKUH-Holter (NCKUHHolter) database. The methodology for establishing these databases has been described and validated in our previous studies (15-17). The study population included all patients who underwent 24-h Holter ECG monitoring between July 1, 2011, and December 31, 2018, at the NCKUH. An indication for Holter ECG monitoring included clinical suspicion of arrhythmia, syncope, and palpitation. With regard to patients who underwent multiple Holter studies, the index Holter study was defined as that with the largest VPC burden. We excluded patients with an incomplete chart review, patients under 18 years of age, patients who had a loss of follow-up or were not regularly followed for over 6 months, and patients who did not complete a Holter study. The clinical data used in this study were obtained from NCKUH with IRB approval (B-ER-108-290), and it was registered to ClinicalTrials.gov (http://clinicaltrials.gov/ ct2/show/NCT03877614).

\section{Clinical Characteristics and Outcome}

Data were collected from the NCKUH-EMR database. This database was excavated from the electrical medical record system of the NCKUH using a data mining technique. Clinical diagnosis from the database was validated by a random sample survey performed by three cardiologists. The accuracy of the sample survey was $99 \%$. Clinical characteristics of interest included age, gender, and history of diseases, including DM, dyslipidemia, hypertension, stroke, $\mathrm{CKD}, \mathrm{HF}$, and cardiovascular disease
(CVD). HF was defined as a left ventricular ejection fraction of $<50 \%$, and CVD was defined as composite diseases, including coronary artery disease, stroke, and peripheral artery disease. The clinical outcomes of interest in this study were all-cause mortality and cardiovascular death. Mortality data were retrieved from the medical records of our hospital.

\section{Ascertainment of Death and Definition of Cardiovascular Death}

The ascertainment of death was performed by data mining and a chart review. As this is a retrospective cohort study enrolling patients with a regular follow-up from 2011 to 2018, the validation of death could be achieved by a chart review. A telephone interview was used for uncertain patients to validate the survival status. The primary cause of death was defined as the underlying disease or injury that initiated the course of events that resulted in death. Cardiovascular death was defined as death attributable to acute myocardial infarction, sudden cardiac death, HF, stroke, cardiovascular procedures, cardiovascular hemorrhage, and death due to any other cardiovascular cause (18). The cause of death for each patient was determined by comprehensive judgment according to the certification of death and discharge diagnosis recorded in the EMR, which was reviewed by a cardiologist (P-TL) and validated by the two other independent cardiologists ( $\mathrm{T}-\mathrm{CH}$ and $\mathrm{M}-\mathrm{HH}$ ).

\section{4-H Holter ECG Variable}

Data were collected from the NCKUH-Holter database as formal Holter reports are contained in this database. VPC was identified by a simultaneous 3-channel 24-h Holter monitoring (NorthEast Monitoring, Inc., DR200/HE Digital Recorder). Holter monitor raw data were edited by the four experienced technicians (Ms. Y.L.Chen, Ms. S.F. Hsu, Ms. C.Y. Tseng, and Ms. Y.S. Hsueh), and all arrhythmic episodes, unknown strips, and final formal Holter reports were reviewed and confirmed by five senior cardiologists. Variables of interest from the Holter data in this study included VPC burden, atrial premature complex (APC) burden, supraventricular tachycardia (SVT), and ventricular tachycardia (VT). Both monomorphic and polymorphic VPCs were included in the study. Incomplete or uninterpretable Holter studies due to the dislodgement of the electrogram patch, machine malfunction, uncooperative patients, or standstill strips were excluded. All valid Holter studies were eligible for further analysis. A pilot validation study was performed to guarantee the accuracy of the database. A total of 200 patients were randomly selected, and four random traits were obtained, such as baseline characteristics, comorbidities, or medications. Each patient was manually reviewed by two of the three cardiovascular physicians (P-TL, T-CH, and $\mathrm{M}-\mathrm{HH}$ ). The accuracy rate of all traits was $99.12 \%(793 / 800)$.

\section{Statistical Analysis}

Baseline clinical characteristics were reported as means \pm SDs for continuous variables, median [interquartile range (IQR)] for non-normal distribution variables, and as percentages for categorical variables. As the daily VPC burden is not a natural distribution, a natural log transformed VPC was created as 


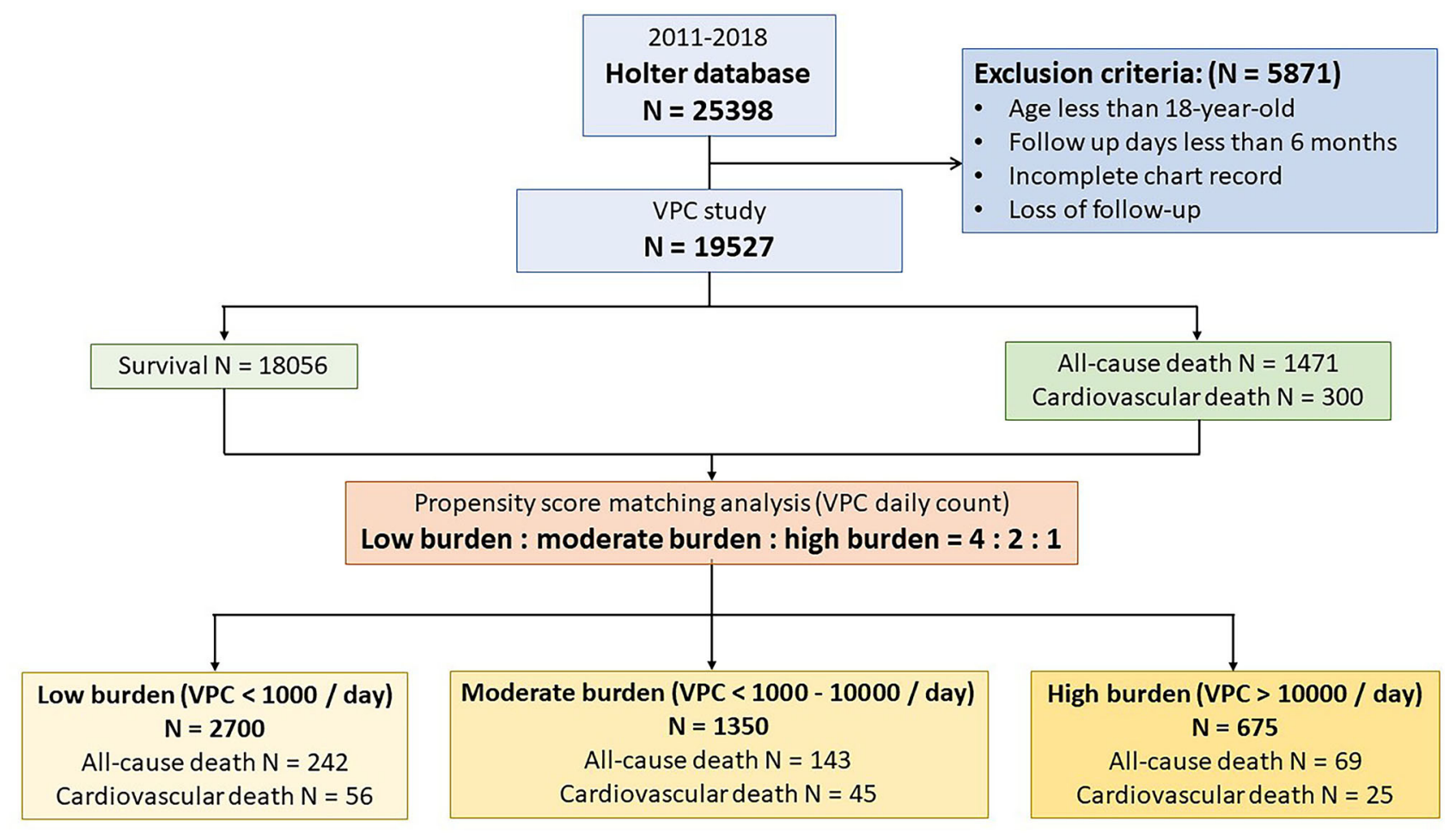

FIGURE 1 | Flow chart of the study protocol. A total of 19,527 patients were enrolled in the study. During the follow-up period, 1,471 patients suffered from death. Cardiovascular death accounts for $20.4 \%(n=300)$ of all-cause death. A propensity score matching analysis was performed to analyze the effect of ventricular premature complex (VPC) burden on all-cause death and cardiovascular death.

a representative continuous variable. Student's $t$-test and Chisquared test with Yates' correction was used to analyze the continuous and categorical variables, respectively. A univariate and multivariate Cox regression model was performed to analyze the contribution of clinical and Holter variables to cardiovascular death. The adjusted variables included age, gender, DM, dyslipidemia, hypertension, CKD, HF, CVD, antiplatelet drugs, AADs, beta blocker, and VT. A propensity score matching method was used to analyze the clinical outcomes (all-cause death and cardiovascular death) of patients with low $(<1,000 /$ day $)$, moderate (1,000-10,000/day), and high $(>10,000)$ VPC burdens. Crude event rates from Kaplan-Meier survival curves were compared between these groups using the logrank test. To further elucidate the contribution of VPC burden to cardiovascular death, cumulative incidence functions (CIFs) were used to estimate the incidence of each of the different types of competing risks. A Fine-Gray subdistribution hazard model was used to estimate the prognosis of VPC in the presence of other competing risks (19).

\section{RESULT}

\section{Baseline Characteristics, VPC Burden, and Clinical Outcome of the Study Cohort}

From 2011 to 2018, we consecutively analyzed 30,488 records of Holter monitoring from 25,398 patients. Patients with an incomplete chart review and who aged less than 18 years and had a loss of follow-up or were followed up for less than 6 months were excluded from this study. About 24,071 Holter monitoring records belonging to 19,527 patients were analyzed. The study design is illustrated in Figure 1. The study population was predominately $52.8 \%$ male with a mean age of $59.6 \pm 17.5$ years, $22.4 \%$ DM, $46.0 \%$ dyslipidemia, $49.3 \%$ hypertension, $8.7 \%$ experienced a stroke, $19.8 \% \mathrm{CKD}, 12.4 \% \mathrm{HF}$, and $22.9 \%$ CVD. In terms of the Holter study, the mean VPC burden was 1,129 \pm $4,422 /$ day, and $6.3 \%$ of the study patients had VT. The VPC count was translated to logarithm due to the right skew distribution. The mean log VPC was $3.0 \pm 2.9$. Non-sustained VT was detected in $6.3 \%$ of the study population. During a mean 955 -day followup, $1,471(7.53 \%)$ patients died. Among the deceased patients, $300(1.53 \%)$ deaths were attributed to cardiovascular death, 1,103 (5.65\%) deaths were attributed to non-cardiovascular death, and the remaining 71 deaths were of an unknown etiology due to death occurring prior to arriving at the hospital or an incomplete medical record.

\section{VPC Burden Is Associated With All-Cause and Cardiovascular Death}

To evaluate whether VPC burden is associated with all-cause death or cardiovascular death, the study cohort was divided into three groups based on the VPC counts, including a low VPC burden defined as $<1,000 /$ day $(n=16,996)$, a moderate VPC burden defined as $1,000-10,000 /$ day $(n=1,856)$, and a high VPC burden defined as $>10,000 /$ day $(n=675)$ based on the previous literature (2). The median and IQR of daily VPC count in the three groups was $5(1-44), 2,712(1,618-4,982)$, and 17,726 
$(13,306-25,704)$. Meanwhile, the median and IQR of daily VPC burden in the three groups was $<0.1 \%(<0.1-<0.1 \%), 2.6 \%(1.5-$ $4.9 \%)$, and $17.5 \%$ (13.1-25.4\%), respectively. In comparison to the low burden group, the moderate and high burden groups had more comorbidities, including hypertension, DM, HF, CVD, and CKD. The prevalence of atrial fibrillation and stroke was high in the moderate burden group but not in the high burden group compared to the low burden group. In terms of the medication history, antiplatelet medications, angiotensin-converting enzyme inhibitor/angiotensin receptor blocker (ACEI/ARBs), and statins were more frequent in the moderate and high burden group. Regarding AADs, the use of class Ib AAD was more frequent in comparison with other type AADs for patients with a high VPC burden. The outcome before the propensity score matching analysis in the three groups showed higher all-cause mortality (VPC burden: low, 7.1\%; moderate, $11.0 \%$; and high, 10.2\%) and cardiovascular mortality (VPC burden: low, 1.3\%; moderate, $3.3 \%$; and high, $3.7 \%$ ) in the moderate and high burden groups in comparison with the low burden group (Table 1).

To validate the effect of VPC burden on all-cause death and cardiovascular death, the daily VPC count was translated to logarithm due to a right skew deviation of the raw VPC daily count, which showed that the VPC burden (log VPC) was significantly higher in the all-cause death population (3.9 \pm 3.0 vs. $2.9 \pm 2.9, p<0.001)$ and cardiovascular death $(5.0 \pm 3.0$ vs. $2.9 \pm 2.9, p<0.001)$ population in comparison with the survival population in this cohort (Supplementary Tables 1, 2). Moreover, the daily burden of the cardiovascular death population was even higher than that of the all-cause death population. To further analyze the effect of the daily VPC burden on cardiovascular death, a Cox regression analysis and a competing risks survival analysis using the Fine-Gray subdistribution hazard model were performed (Figure 3 and Supplementary Table 3). In comparison with the low burden group, the moderate burden group showed a higher risk of cardiovascular death (multivariable Cox regression model adjusted hazard ratio (HR) 1.47, 1.09-1.98, $p<0.05$, Fine and Gray's competing risk model adjusted HR 1.48, 1.09-2.01, $p<$ 0.05). The trend was even higher in the high burden group (Cox regression model adjusted $\mathrm{HR} 1.68,1.07-2.63, p<0.05$, Fine and Gray's competing risk model adjusted HR 1.70, 1.06-2.71 $p<0.05)$. These results indicated a moderate and high VPC burden led to more cardiovascular death. Regarding the all-cause death, neither the moderate burden group (multivariable Cox regression model adjusted HR 1.14, 0.98-1.33, $p=0.11$ ) nor the high burden group (multivariable Cox regression model adjusted HR $1.10,0.85-1.42, p=0.16$ ) showed a significantly higher risk in comparison with the low burden group.

\section{High Burden of VPC Led to More Cardiovascular Death Confirmed by Propensity Score Match Analysis and Kaplan-Meier Analysis}

To evaluate the prognostic effect of VPC burden on all-cause death and cardiovascular death, we tried to use the Kaplan-Meier analysis. The analysis demonstrated that a high VPC burden led
TABLE 1 | Baseline characteristics, medication history, Holter data, and clinical outcome based on different VPC burdens.

\begin{tabular}{ccc}
\hline $\begin{array}{c}\text { Low burden }(N \\
=16996)\end{array}$ & $\begin{array}{c}\text { Moderate } \\
\text { burden } \\
(N=1856)\end{array}$ & $\begin{array}{c}\text { High burden }(N) \\
=675)\end{array}$
\end{tabular}

\begin{tabular}{|c|c|c|c|c|}
\hline \multicolumn{5}{|c|}{ Clinical characteristics } \\
\hline Age (y) & $59.2 \pm 17.8$ & $62.9 \pm 16.1$ & $60.9 \pm 16.4$ & $<0.001$ \\
\hline Male & $7,793(45.9)$ & $1,048(56.5)$ & $380(56.3)$ & $<0.001$ \\
\hline Follow-up days & $987(552-1428)$ & $\begin{array}{c}973 \\
(527-1436)\end{array}$ & $963(541-1441)$ & 0.901 \\
\hline HTN & $8,230(48.4)$ & $1038(55.9)$ & $352(52.2)$ & $<0.001$ \\
\hline DM & $3,653(21.5)$ & $520(28.0)$ & $194(28.7)$ & $<0.001$ \\
\hline Dyslipidemia & $7,679(45.2)$ & $972(52.4)$ & $330(48.9)$ & $<0.001$ \\
\hline $\mathrm{HF}$ & $1,824(10.7)$ & $440(23.7)$ & $160(23.7)$ & $<0.001$ \\
\hline CVD & $3,640(21.4)$ & $632(34.1)$ & $191(28.2)$ & $<0.001$ \\
\hline CAD & $1,841(10.8)$ & $374(20.2)$ & $120(17.8)$ & $<0.001$ \\
\hline Stroke & $1,437(8.5)$ & $206(11.1)$ & $54(8.0)$ & 0.001 \\
\hline PAD & $362(2.1)$ & $52(2.8)$ & $17(2.5)$ & 0.020 \\
\hline AF & 2,899 (17.1) & $477(25.7)$ & $121(17.9)$ & $<0.001$ \\
\hline CKD & $3,197(18.8)$ & $499(26.9)$ & $170(25.2)$ & $<0.001$ \\
\hline \multicolumn{5}{|l|}{ Medication } \\
\hline Aspirin & 3,461 (20.4) & $485(26.1)$ & $146(21.6)$ & $<0.001$ \\
\hline$P_{2} Y_{12}$ inhibitor & $1,350(7.9)$ & $244(13.2)$ & $82(12.2)$ & $<0.001$ \\
\hline ACEi/ARB & 2,697 (15.9) & $455(24.5)$ & $156(23.1)$ & $<0.001$ \\
\hline Statin & 3,059 (18.0) & $414(22.3)$ & 139 (20.6) & $<0.001$ \\
\hline Class la AAD & $7(0.04)$ & $1(0.05)$ & $0(0)$ & 0.839 \\
\hline Class Ib AAD & 367 (2.2) & $372(21.0)$ & 254 (39.6) & $<0.001$ \\
\hline Class Ic AAD & $317(1.9)$ & $42(2.3)$ & $17(2.5)$ & 0.259 \\
\hline Class III AAD & $903(5.3)$ & $236(12.7)$ & $117(17.3)$ & $<0.001$ \\
\hline Beta blocker & $3,478(20.5)$ & $484(26.1)$ & $161(23.9)$ & $<0.001$ \\
\hline Non-DHP CCB & $873(5.1)$ & $112(6.0)$ & $61(9.0)$ & $<0.001$ \\
\hline \multicolumn{5}{|l|}{ Holter data } \\
\hline VPC count & $5(1-44)$ & $\begin{array}{c}2,712 \\
(1,618-4,982)\end{array}$ & $\begin{array}{c}17,726 \\
(13,306-25,704)\end{array}$ & $<0.001$ \\
\hline VPC burden (\%) & $\begin{array}{c}<0.1 \\
(<0.1-<0.1)\end{array}$ & $2.6(1.5-4.9)$ & $17.5(13.1-25.4)$ & $<0.001$ \\
\hline VT & $544(3.2)$ & $418(22.5)$ & $263(38.9)$ & $<0.001$ \\
\hline APC count & $17(3-104)$ & $32(3-566)$ & $15(2-107)$ & $<0.001$ \\
\hline \multicolumn{5}{|l|}{ Clinical outcome } \\
\hline All-cause death & $1,198(7.1)$ & $204(11.0)$ & 69 (10.2) & $<0.001$ \\
\hline CV death & $214(1.3)$ & $61(3.3)$ & $25(3.7)$ & $<0.001$ \\
\hline
\end{tabular}

$A A D$, antiarrhythmic drug; $A C E / A R B$, angiotensin-converting enzyme inhibitor/angiotensin receptor blocker; $A F$, atrial fibrillation; $A P C$, atrial premature complex; CKD, chronic kidney disease; CV death, cardiovascular death; CVD, cardiovascular disease (including coronary artery disease, stroke, and peripheral artery disease); DM, diabetes mellitus; HF, heart failure; HTN, hypertension; IQR, interquartile range; non-DHP CCB, non-dihydropyridine calcium channel blocker; VPC, ventricular premature complex. VT, ventricular tachycardia. Age was shown as mean $\pm S D$. Follow-up days, VPC count, VPC burden, and APC count were shown as median (IQR). The other variables were shown as $n(\%)$.

to more all-cause death $(\log \operatorname{rank} p<0.001)$ and cardiovascular death $(\log \operatorname{rank} p<0.001)$ in comparison with a low burden (Figures 2A,B). However, clinical preexisting diseases had an 


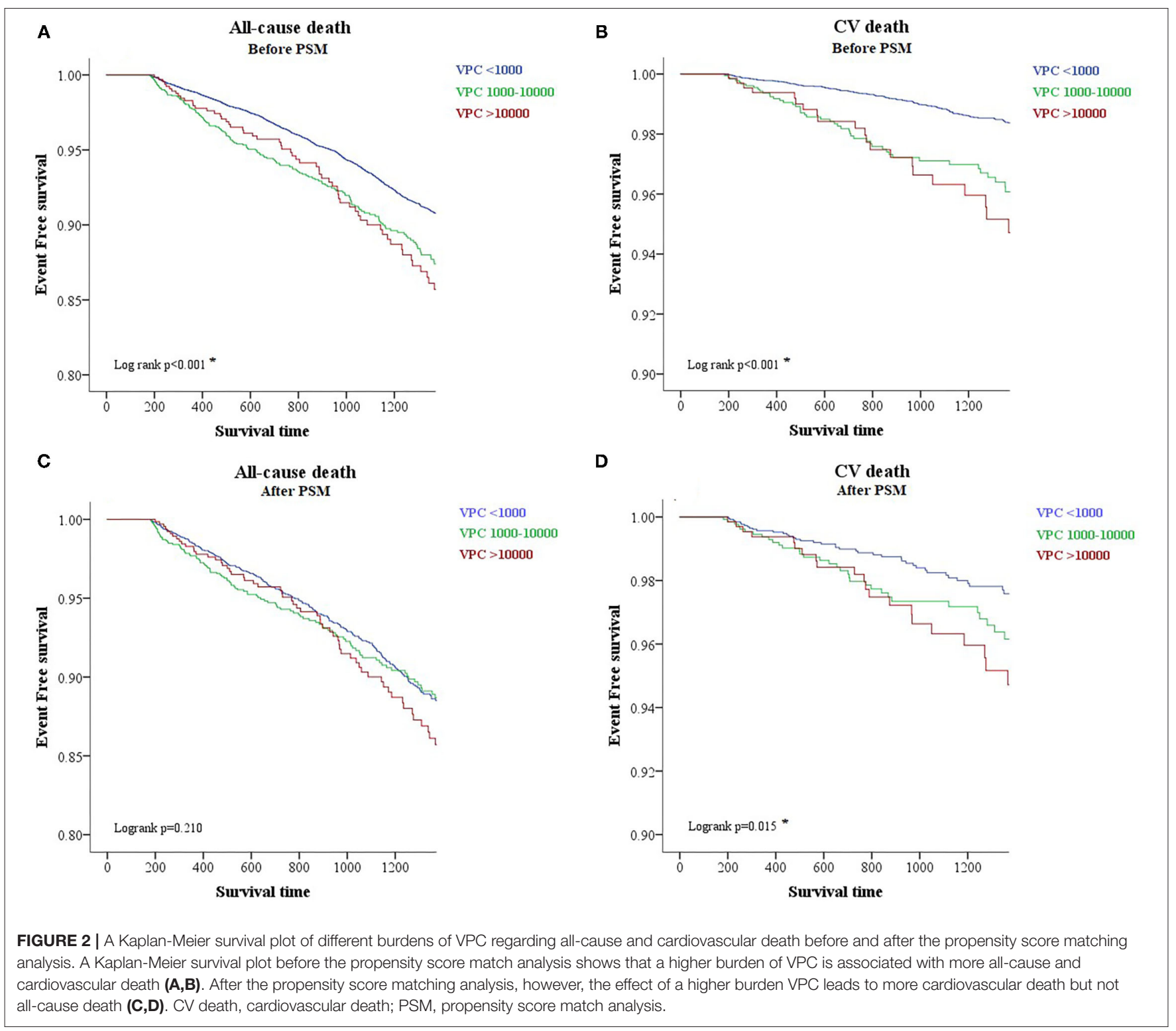

impact on the outcome of all-cause and cardiovascular deaths, including age, gender, hypertension, DM, stroke, CKD, HF, and CVD. To elucidate whether a high and moderate VPC burden led to all-cause and cardiovascular mortality, a propensity score matching method was applied in this cohort study. Three individual groups were divided by the previous definitions and propensity score matched with a 4:2:1 ratio (Table 2). All-cause mortality was not different among the three groups (low, 9.0\%; moderate, $10.6 \%$; and high, $10.2 \%, p=0.2129)$; however, both moderate and high VPC burdens led to more cardiovascular death in comparison with a low VPC burden (low, 2.1\%; moderate, 3.3\%; and high, 3.7\%, $p=0.0125$ ) (Table 2). The Kaplan-Meier analysis after a propensity score matching analysis also demonstrated the same trend. All-cause death did not show a statistical significance in these three groups $(\log \operatorname{rank} p=$ 0.210 , Figure $2 \mathrm{C}$ ). On the other hand, the moderate and high burden groups showed a significant increase in cardiovascular death in comparison to the low burden group (log $\operatorname{rank} p=$ 0.015 , Figure 2D). Based on the above statistical analyses, a moderate and high VPC burden led to more cardiovascular death compared with a low VPC burden.

\section{Subgroup Analysis of Different Burdens of VPC and Cardiovascular Death}

The subgroup analysis of the adjusted HRs for cardiovascular death compared the high burden group or moderate burden group with the low burden group (Figure 4). In terms of baseline characteristics, elderly patients ( $\geq 60$ years old) with an underlying history of HF and hypertension, and patients who did not use antiplatelet drugs, beta blockers, classes Ib and III AADs, or non-dihydropyridine calcium channel blockers (nonDHP CCB) had a higher risk of cardiovascular death in the high 


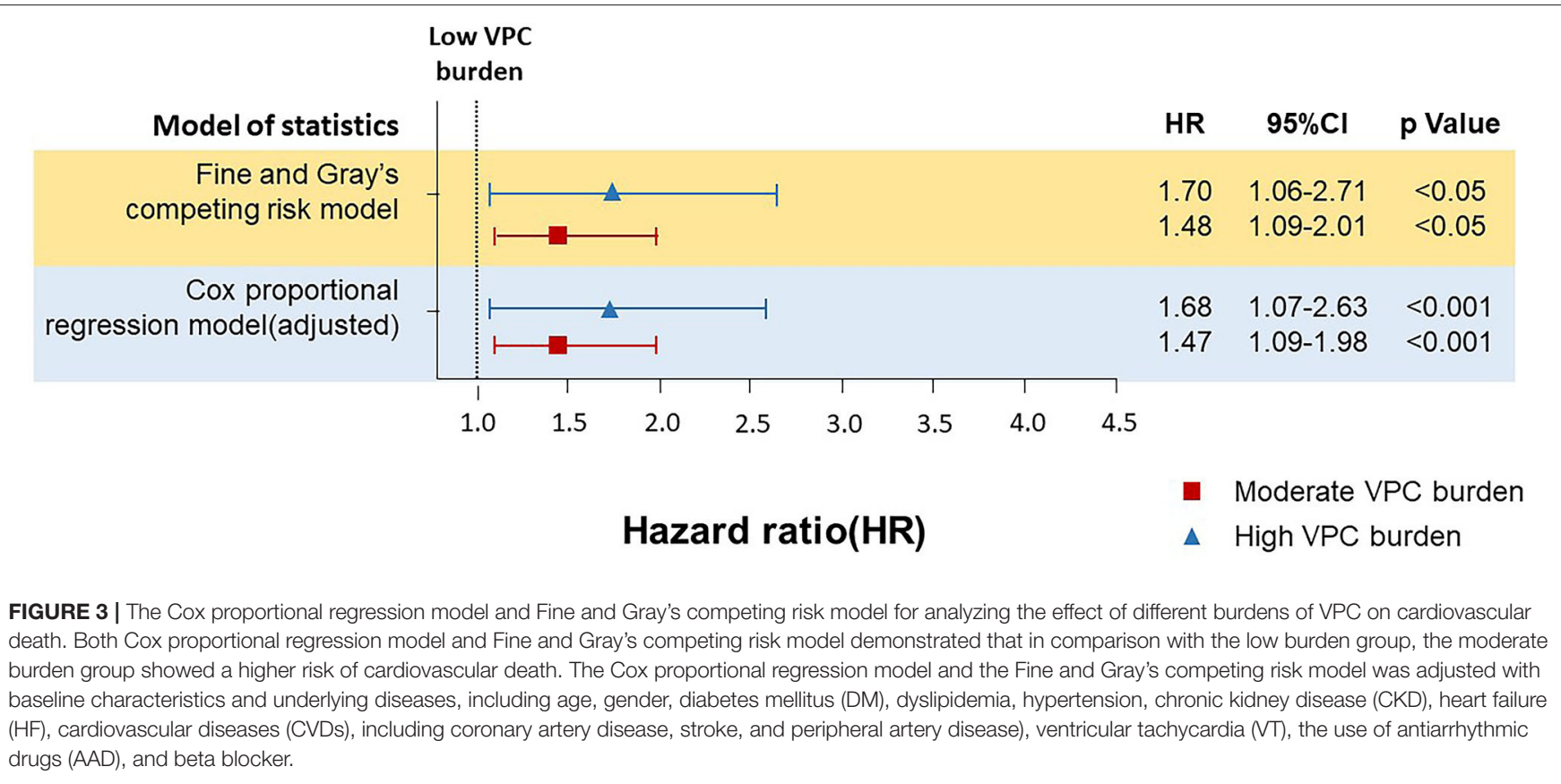

TABLE 2 | Clinical outcome after the propensity score matched group analysis for a low, moderate, and high burden of VPC (4:2:1 match).

\begin{tabular}{|c|c|c|c|c|}
\hline & Low $(N=2,700)$ & $\begin{array}{c}\text { Moderate }(N= \\
1,350)\end{array}$ & High $(N=675)$ & \multirow[t]{2}{*}{$P$ value } \\
\hline & $\begin{array}{c}\text { Mean (SD)/N } \\
(\%)\end{array}$ & $\begin{array}{c}\text { Mean (SD)/N } \\
(\%)\end{array}$ & $\begin{array}{c}\text { Mean (SD)/N } \\
(\%)\end{array}$ & \\
\hline Age & $59.9(16.7)$ & $61.1(16.5)$ & 60.9 (16.4) & 0.090 \\
\hline Male & 1,492 (55.3) & 754 (55.9) & 380 (56.3) & 0.864 \\
\hline DM & 753 (27.9) & $389(28.8)$ & $194(28.7)$ & 0.793 \\
\hline HTN & $1,379(51.1)$ & 707 (52.4) & 352 (52.2) & 0.704 \\
\hline CKD & 648 (24.0) & 341 (25.3) & $170(25.2)$ & 0.621 \\
\hline $\mathrm{HF}$ & $660(24.4)$ & 315 (23.3) & $160(23.7)$ & 0.722 \\
\hline CVD & 499 (18.5) & 248 (18.4) & $127(18.8)$ & 0.970 \\
\hline All-cause death & $242(9.0)$ & 143 (10.6) & 69 (10.2) & 0.213 \\
\hline CV death & $56(2.1)$ & $45(3.3)$ & $25(3.7)$ & 0.013 \\
\hline
\end{tabular}

CKD, chronic kidney disease; CV death, cardiovascular death; CVD, cardiovascular disease (including coronary artery disease, stroke, and peripheral artery disease); DM, diabetes mellitus; HF, heart failure; HTN, hypertension; VPC, ventricular premature complex. Age was shown as mean (SD), and the other variables were shown as $n(\%)$. Bold value means statistically significant $(p<0.05)$.

burden group. In the moderate burden group, a similar trend was also observed. We conclude that elderly patients with underlying diseases, and those who do not use AADs, had a higher risk of cardiovascular death with moderate or high VPC burdens.

\section{DISCUSSION}

In a population-based cohort study of 19,527 patients who underwent 24-h Holter ECG monitoring in Taiwan, a high and moderate VPC burden was associated with a high risk of cardiovascular death. Moreover, in the high and moderate burden groups, patients without the use of cardioprotective drug $s$ and AADs had higher risks of cardiovascular death.

\section{VPC and Cardiovascular Mortality}

Previous cohort studies of VPC have shown that the frequent VPC was associated with increased all-cause mortality. In a previous study, which contained 5,778 patients with a mean follow-up period of 10 years, Lin et al. postulated that a cut-off value of 12 VPCs per day was predictive of all-cause mortality with an area under the receiver operating characteristics (ROC) curve of $59.6 \%$ (1). Another community-based study, which enrolled 15,792 patients, also demonstrated that the presence of VPC had a higher rate of subsequent $\mathrm{HF}$ as compared to no VPC on a 2-min rhythm ECG strip over an average follow-up of 15.6 years (20). However, the abovementioned two studies did not mention cardiovascular mortality. A recent subgroup analysis from the CHF-STAT study involving 421 patients reported a group of VPC-cardiomyopathy with a median count of 2,832 VPCs per day, which was associated with more cardiac death and resuscitated cardiac arrest (10). In our study, we collected 19,527 patients with continuous electrogram monitoring (Holter), and we also support that VPC burden of more than 1,000/day was associated with more cardiovascular death, which was consolidated by the propensity score match analysis. Furthermore, the relationship between VPC and cardiovascular death was tested by the univariate and multivariate Cox proportional hazards model and the Fine-Gray subdistribution hazard model (19, 2123). In the field of cardiovascular research regarding disease incidence and prognosis prediction, the performance of the FineGray subdistribution hazard model prevented overestimating the incidence of the outcome of interest when the competing 


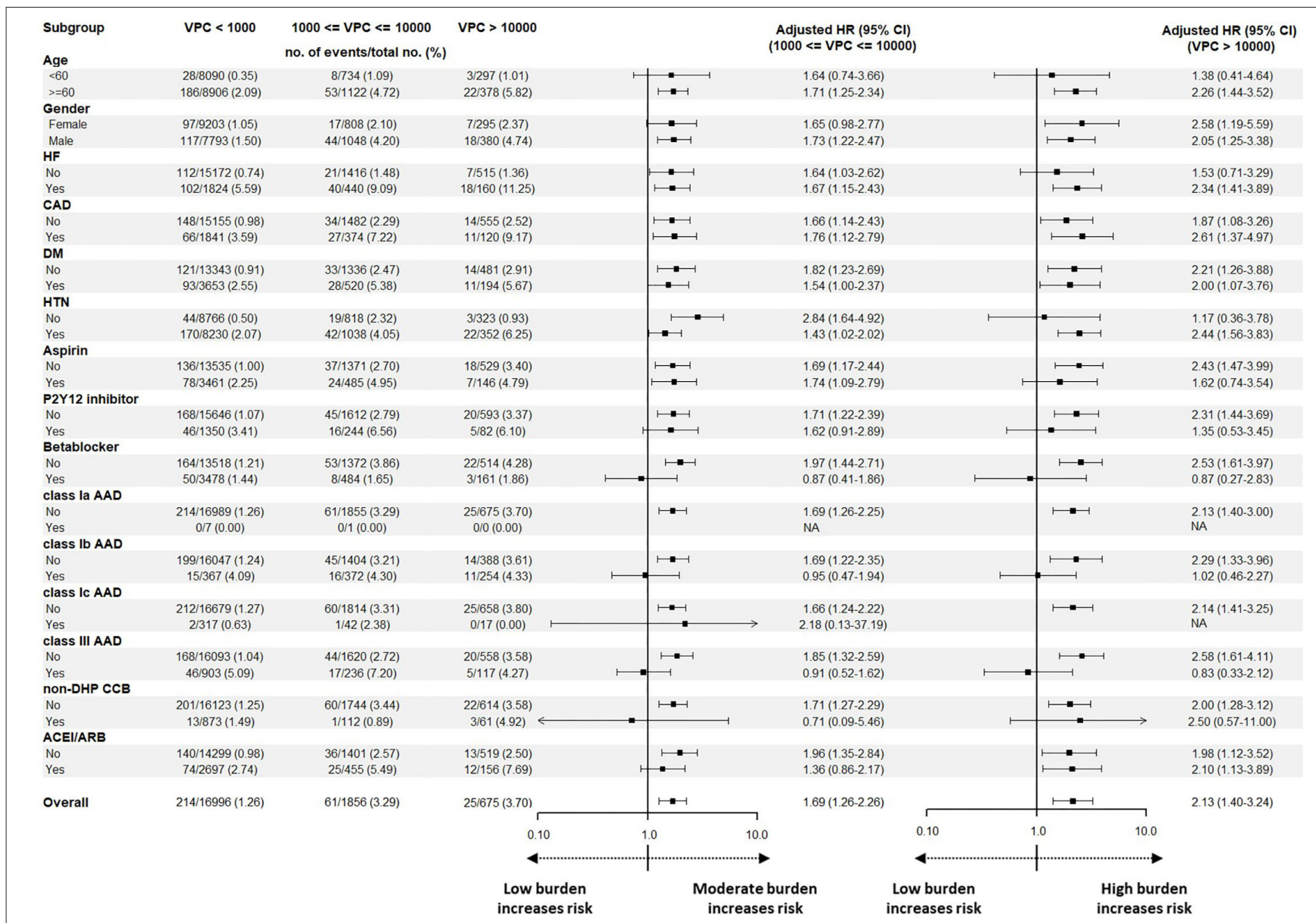

FIGURE 4 | A subgroup analysis before the propensity match analysis for analyzing the effect of baseline characteristics, underlying disease, and medication on cardiovascular death. Illustration of the subgroup analysis before the propensity score match analysis. Male, elderly, patients without hypertension or DM, and patients who did not use P2Y12 inhibitor, beta blocker, non-DHP CCB, ACEl/ARB, or AADs showed an increased risk to have cardiovascular death in comparison with the low burden group. Regarding the high burden group, the elderly, patients with HF and hypertension, and patients who did not use aspirin, beta blockers, non-DHP CCB, or AADs showed an increased risk to have cardiovascular death in comparison to the low burden group. AAD, antiarrhythmic drug; $A C E I / A R B$, angiotensin-converting enzyme inhibitor/angiotensin receptor blocker; CAD, coronary artery disease; Cl, confidence interval; DM, diabetes mellitus; HF, heart failure; HTN, hypertension; non-DHP CCB, non-dihydropyridine calcium channel blocker.

risk was present (19). All these models yielded similar results. The abovementioned finding supports the hypothesis that VPC burden had a negative impact on cardiovascular mortality.

\section{Possible Mechanisms of VPC-Induced Cardiovascular Mortality}

Besides the cohort studies, we postulated several possible mechanisms of VPC-induced cardiovascular death. First, a high VPC burden is associated with cardiomyopathy. The effect of premature contraction on the cardiac structural change was shown to be limited to the VPC, and the same situation did not appear in the atrial premature contraction (24). To demonstrate the ventricular structural change induced by VPC, a study of programmed pacemaker stimulation simulating persistent VPC in the canine models can induce functional changes in the myocardium and lead to a significant reduction of the left ventricular systolic function (25). Previous animal models also showed that left ventricular dys-synchrony and eccentric hypertrophy caused by VPC are also considered to be associated with the deterioration of the left ventricular systolic function $(26,27)$. Another swine model of VPC-induced cardiomyopathy model further demonstrated persistent changes in myocardial fibrosis and left ventricular dys-synchrony after the elimination of VPC (28). Left ventricular fibrosis and dys-synchrony caused by VPC were proven to be associated with the worse cardiovascular outcome $(29,30)$. The above studies provided insights that VPC made a significant impact on left ventricular functional and structural dysfunction and potentially led to more cardiac death. Second, electrical remodeling also has an important role. An animal and a cellular study proposed that the myocardial cells extracted from a chronic frequent VPC canine model can induce a heterogeneous reduction in Ito, IK1, 

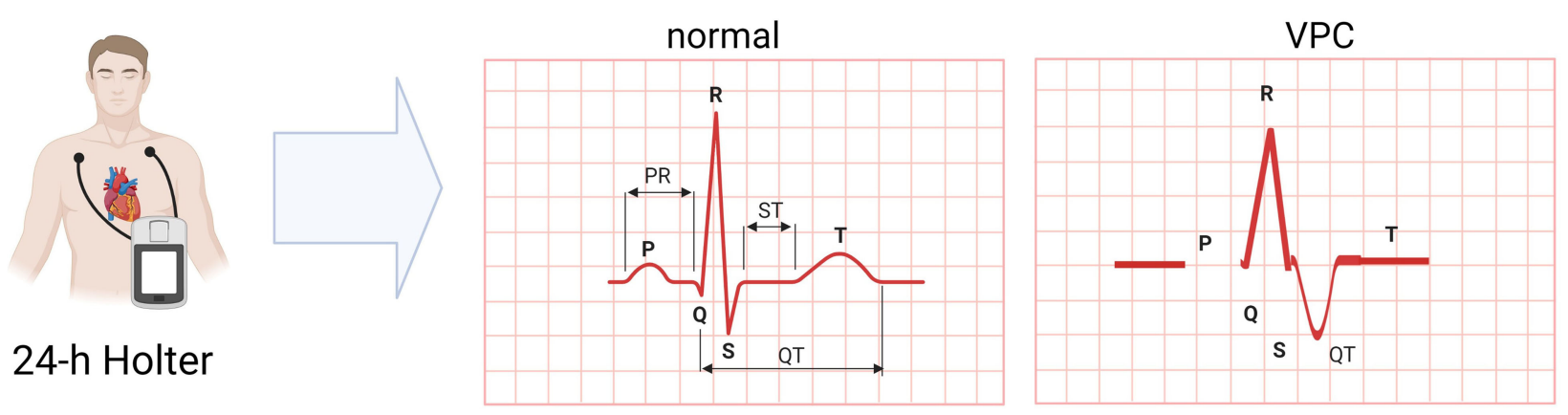

\section{VPC patients}

\section{Propensity score matched analysis}

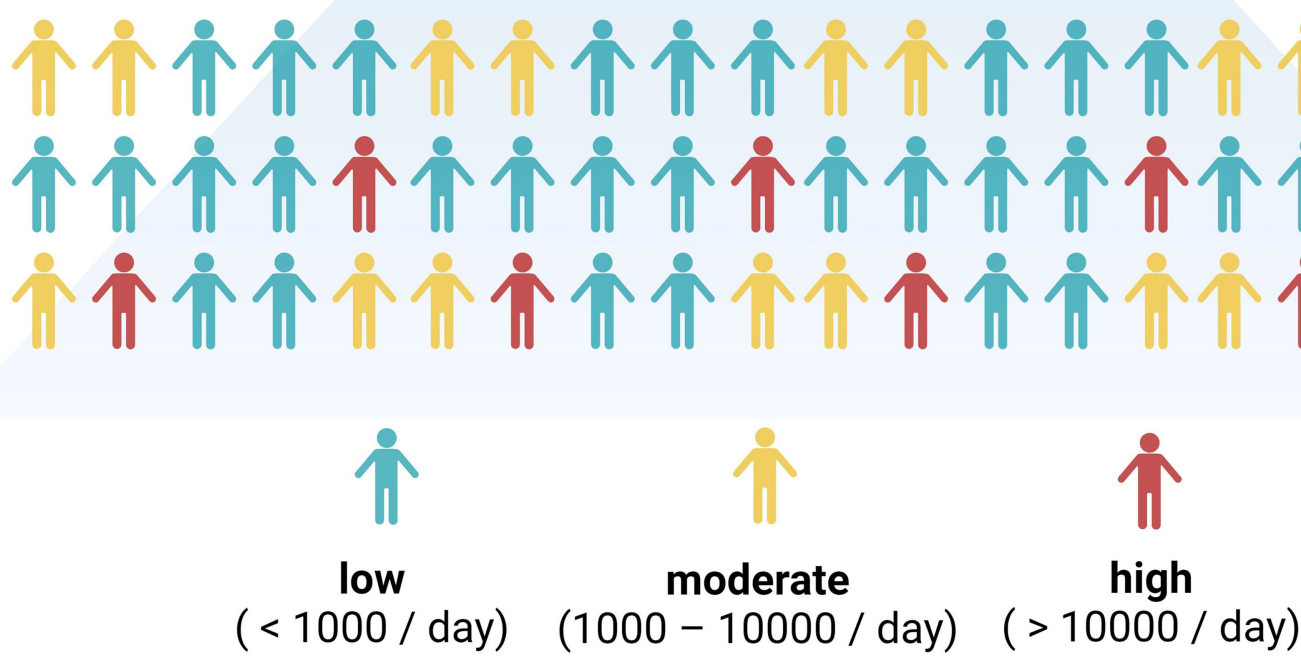

\section{VPC burden}

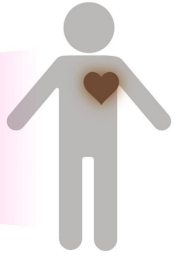

\section{CV death $\quad \uparrow 1.48$ times $\quad \uparrow 1.70$ times}

FIGURE 5 | Graphic abstract of this study. A high and moderate VPC burden led to more cardiovascular death, and a high VPC burden had 1.70 times of cardiovascular death risk.

and ICaL. The increased repolarization heterogeneity due to decreased outward and inward L-type calcium currents were associated with significant beat-to-beat action potential duration dispersion and may result in an increased risk of reentrant ventricular tachyarrhythmia, which is related to sudden cardiac death (31). Third, VPC can also induce cardiac autonomic dysregulation. VPC delivery in both human and porcine models elicited a significant cardiac sympathetic response than other stimuli $(32,33)$. In addition, VPC-induced sympathetic neural hyperactivity persisted despite the withdrawal of electrical stimulation and normalization of left ventricular ejection fraction (34). Cardiac autonomic dysregulation is an important trigger and substrate for ventricular pro-arrhythmia, leading to cardiac mortality. 


\section{Risk Stratification of VPC in Cardiovascular Death}

Recently, Voskoboinik et al. postulated an "ABC-VT" scoring system to evaluate the adverse outcome in a frequent VPC cohort including 206 patients. The ABC-VT score includes a superiorly directed VPC axis, a high VPC burden of $10-20 \%$ and $>20 \%$, a VPC coupling interval $>500 \mathrm{~ms}$, and the presence of nonsustained VT. Patients with frequent VPC and ABC-VT score $>4$ had a higher risk to have composite endpoints of a left ventricular ejection fraction decline by $10 \%$, HF hospitalization, or cardiovascular mortality. In this study, the VPC burden of 10$20 \%$ and $>20 \%$ had an odds ratio of 3.5 and 4.4 , respectively, to meet the composite endpoints (35). In terms of our current study, the most significant advantage was the number of patients. Regarding the number of patients with a high VPC burden, we have 650 patients, which was three times more than the previous study. Moreover, the cohort study was large enough to arrange a comparison between a moderate VPC burden and a low VPC burden with a 1:2:4 ratio of propensity score match analysis. Our study demonstrated that not only the high VPC burden group but also the moderate VPC burden group experienced a higher risk of cardiovascular mortality than the low VPC burden group ( 3.7 vs. 3.3 vs. $2.1 \%$, respectively; $p=0.0125$ ) after the propensity score match analysis. Currently, there was no study that evaluated the effect of moderate VPC burden on long-term prognosis. In our opinion, medical treatment should be taken into consideration for patients with a moderate VPC burden who still had a higher risk of cardiovascular death compared with the low VPC burden group.

\section{Possible Risk Factors of High Burden VPC Associated With Cardiovascular Death}

Heart failure patients with a high VPC burden were reported to be at a higher risk of cardiovascular events (36). In the subgroup analysis of the current study, not only HF but also hypertension and coronary artery disease contributed to a higher risk of cardiovascular death in the high VPC burden group. Furthermore, according to a previous study, the treatment to VPC was able to effectively reduce the VPC burden and prevent the decline of the left ventricular systolic function (37). In the subgroup analysis of our study, the use of cardioprotective drugs, including antiplatelet drugs, beta blockers, AADs, and non-DHP $\mathrm{CCB}$, in the high VPC burden group is associated with less cardiovascular death. Although class Ic AADs were reported to be effective in the treatment of VPC-induced cardiomyopathy, the effect of other AADs, including mexiletine and amiodarone, beta blockers, and non-DHP CCB, were not reported to reduce cardiovascular death in high VPC burden patients in the literature (9). Comprehensive prospective studies were needed to clarify whether these drugs were effective for preventing cardiovascular death in patients with a high VPC burden.

\section{Limitations of This Study}

Several limitations should be mentioned. First, this was a singlehospital, retrospective study based on a chart review. Although incomplete chart records or the loss of follow-up had been excluded from the study, the impact of the excluded cases on outcomes is unknown. Second, the study cohort was selected from a single center in Southern Taiwan. The populations included in the current study were mostly Asians. Whether our results could be applied to other races remained unknown. Third, only patients who were followed up more than 180 days were enrolled in the study. However, the median follow-up period of the study was only 955 days, which may not be long enough to trace the cardiovascular death although a competing risk analysis model was used to fix the disadvantage. Fourth, serial echocardiography data were not available during data mining, as a result, the VPC-induced cardiomyopathy or VPC-induced HF cannot be defined clearly and should not be mentioned in the current study. Finally, interventional data, including coronary intervention and catheter ablation, were not available during data mining. The effect of cardiovascular intervention to reduce mortality remained unknown.

\section{CONCLUSION}

A higher VPC burden, particularly those with a high ( $>10,000 /$ day) or moderate $(1,000-10,000 /$ day $)$ burden, is an independent risk factor of cardiovascular mortality. Patients in the high VPC burden group were at a higher risk of experiencing cardiovascular death (Figure 5).

\section{DATA AVAILABILITY STATEMENT}

The data analyzed in this study is subject to the following licenses/restrictions: The data reported in this study cannot be deposited in a public repository because it was from the NCKUHEMR database, and it did not open to the public. Requests to access these datasets should be directed to Ping-Yen Liu, larry@mail.ncku.edu.tw.

\section{ETHICS STATEMENT}

The studies involving human participants were reviewed and approved by National Cheng Kung University Hospital. Written informed consent for participation was not required for this study in accordance with the national legislation and the institutional requirements.

\section{AUTHOR CONTRIBUTIONS}

P-TL and T-CH: conceptualization. M-HHua: data curation. M-HHua, P-FS, and M-HHun: formal analysis and methodology. P-YL: funding acquisition and supervision. P-TL and P-YL: investigation. P-TL, T-CH, M-HHua, Y-WL, and P-YL: project administration. M-HHua and M-HHun: software. P-TL: writing-original draft. L-WH and P-YL: writing-review and editing. All authors contributed to the article and approved the submitted version.

\section{FUNDING}

This study was funded by National Cheng Kung University Hospital, Tainan, Taiwan (Nos. NCKUH-11104046 and NCKUH-10902023), National Cheng-Kung University for 
its grant (Nos. MOST-109-2634-F-006-023 and MOST-1102634-F-006-020), and Ministry of Science and Technology of Taiwan.

\section{ACKNOWLEDGMENTS}

We would like to thank the National Cheng-Kung University hospital AI capstone team for their bioinformatic technical support. We are also grateful to Dr. Sheng-Hsiang Lin and Ms.
Chih-Hui Hsu for providing the statistical consulting services from the Biostatistics Consulting Center, Clinical Medicine Research Center, NCKUH.

\section{SUPPLEMENTARY MATERIAL}

The Supplementary Material for this article can be found online at: https://www.frontiersin.org/articles/10.3389/fcvm. 2021.797976/full\#supplementary-material

\section{REFERENCES}

1. Lin CY, Chang SL, Lin YJ, Chen YY, Lo LW, Hu YF, et al. An observational study on the effect of premature ventricular complex burden on long-term outcome. Medicine. (2017) 96:e5476. doi: 10.1097/MD.0000000000005476

2. Lee YH, Zhong L, Roger VL, Asirvatham SJ, Shen WK, Slusser JP, et al. Frequency, origin, and outcome of ventricular premature complexes in patients with or without heart diseases. Am J Cardiol. (2014) 114:13738. doi: 10.1016/j.amjcard.2014.07.072

3. Dukes JW, Dewland TA, Vittinghoff E, Mandyam MC, Heckbert SR, Siscovick DS, et al. Ventricular ectopy as a predictor of heart failure and death. J Am Coll Cardiol. (2015) 66:101-9. doi: 10.1016/j.jacc.2015.04.062

4. Kang JW, Yang WH, Chi JE, Chen WT. Higher ventricular premature complex burden is associated with lower systolic blood pressure response. Acta Cardiologica Sinica. (2018) 34:152-8. doi: 10.6515/ACS.201803_34(2).20171117A

5. Kanei Y, Friedman M, Ogawa N, Hanon S, Lam P, Schweitzer P. Frequent premature ventricular complexes originating from the right ventricular outflow tract are associated with left ventricular dysfunction. Ann Noninvas Electrocardiol. (2008) 13:81-5. doi: 10.1111/j.1542-474X.2007.00204.x

6. Latchamsetty R, Yokokawa M, Morady F, Kim HM, Mathew S, Tilz R, et al. Multicenter outcomes for catheter ablation of idiopathic premature ventricular complexes. JACC Clinical electrophysiology. (2015) 1:11623. doi: 10.1016/j.jacep.2015.04.005

7. Sadron Blaye-Felice M, Hamon D, Sacher F, Pascale P, Rollin A, Bongard $\mathrm{V}$, et al. Reversal of left ventricular dysfunction after ablation of premature ventricular contractions related parameters, paradoxes and exceptions to the rule. Int J Cardiol. (2016) 222:31-36. doi: 10.1016/j.ijcard.2016.07.005

8. Fichtner S, Senges J, Hochadel M, Tilz R, Willems S, Eckardt L, et al. Safety and efficacy in ablation of premature ventricular contraction: data from the German ablation registry. Clin Res Cardiol. (2017) 106:4957. doi: 10.1007/s00392-016-1022-9

9. Hyman MC, Mustin D, Supple G, Schaller RD, Santangeli P, Arkles J, et al. Class IC antiarrhythmic drugs for suspected premature ventricular contraction-induced cardiomyopathy. Heart Rhythm. (2018) 15:159-63. doi: 10.1016/j.hrthm.2017.12.018

10. Huizar JF, Fisher SG, Ramsey FV, Kaszala K, Tan AY, Moore H, et al. Outcomes of premature ventricular contraction-cardiomyopathy in the veteran population: a secondary analysis of the CHF-STAT study. JACC Clin Electrophysiol. (2021) 7:380-90. doi: 10.1016/j.jacep.2020.08.028

11. Berruezo A, Penela D, Jáuregui B, Soto-Iglesias D, Aguinaga L, Ordóñez A, et al. Mortality and morbidity reduction after frequent premature ventricular complexes ablation in patients with left ventricular systolic dysfunction. Europace: Eur Pacing, Arrhythm Cardiac Electrophysiol. (2019) 21:107987. doi: 10.1093/europace/euz027

12. Lin CY, Chang SL, Lin YJ, Lo LW, Chung FP, Chen YY, et al. Longterm outcome of multiform premature ventricular complexes in structurally normal heart. Int J Cardiol. (2015) 180:80-5. doi: 10.1016/j.ijcard.2014.11.110

13. Ataklte F, Erqou S, Laukkanen J, Kaptoge S. Meta-analysis of ventricular premature complexes and their relation to cardiac mortality in general populations. Am J Cardiol. (2013) 112:126370. doi: 10.1016/j.amjcard.2013.05.065

14. Jouven $\mathrm{X}$, Zureik $\mathrm{M}$, Desnos $\mathrm{M}$, Courbon $\mathrm{D}$, Ducimetière $\mathrm{P}$. Long-term outcome in asymptomatic men with exercise-induced

premature ventricular depolarizations. $N$ Engl J Med. (2000) 343:826-33. doi: 10.1056/NEJM200009213431201

15. Hsu LW, Chen PW, Chang WT, Lee WH, Liu PY. The role of ROCK in platelet-monocyte collaborative induction of thromboinflammation during acute coronary syndrome. Thromb Haemost. (2020) 120:141731. doi: $10.1055 / \mathrm{s}-0040-1714278$

16. Huang MS, Wang CS, Chiang JH, Liu PY, Tsai WC. Automated recognition of regional wall motion abnormalities through deep neural network interpretation of transthoracic echocardiography. Circulation. (2020) 142:1510-20. doi: 10.1161/CIRCULATIONAHA.120.047530

17. Huang TC, Lee PT, Huang MS, Su PF, Liu PY. Higher premature atrial complex burden from the Holter examination predicts poor cardiovascular outcome. Sci Rep. (2021) 11:12198. doi: 10.1038/s41598-021-9 1800-4

18. Hicks KA, Mahaffey KW, Mehran R, Nissen SE, Wiviott SD, Dunn B, et al. 2017 Cardiovascular and stroke endpoint definitions for clinical trials. J Am Coll Cardiol. (2018) 71:1021-34.

19. Austin PC, Lee DS, Fine JP. Introduction to the analysis of survival data in the presence of competing risks. Circulation. (2016) 133:6019. doi: 10.1161/CIRCULATIONAHA.115.017719

20. Agarwal SK, Simpson RJ, Rautaharju P, Alonso A, Shahar E, Massing M, et al. Relation of ventricular premature complexes to heart failure (from the Atherosclerosis Risk In Communities [ARIC] Study). Am J Cardiol. (2012) 109:105-9. doi: 10.1016/j.amjcard.2011.08.009

21. Lau B, Cole SR, Gange SJ. Competing risk regression models for epidemiologic data. Am J Epidemiol. (2009) 170:244-56. doi: 10.1093/aje/kwp107

22. Austin PC, Lee DS, D'Agostino RB, Fine JP. Developing points-based riskscoring systems in the presence of competing risks. Stat Med. (2016) 35:405672. doi: 10.1002/sim.6994

23. Austin PC, Fine JP. Practical recommendations for reporting Fine-Gray model analyses for competing risk data. Stat Med. (2017) 36:4391400. doi: 10.1002/sim.7501

24. Gunda S, Akyeampong D, Gomez-Arroyo J, Jovin DG, Kowlgi NG, Kaszala $\mathrm{K}$, et al. Consequences of chronic frequent premature atrial contractions: Association with cardiac arrhythmias and cardiac structural changes. $J$ Cardiovasc Electrophysiol. (2019) 30:1952-9. doi: 10.1111/jce.14067

25. Huizar JF, Kaszala K, Potfay J, Minisi AJ, Lesnefsky EJ, Abbate A, et al Left ventricular systolic dysfunction induced by ventricular ectopy: a novel model for premature ventricular contraction-induced cardiomyopathy. Circ Arrhythm Electrophysiol. (2011) 4:543-9. doi: 10.1161/CIRCEP.111. 962381

26. Potfay J, Kaszala K, Tan AY, Sima AP, Gorcsan J, Ellenbogen KA, et al Abnormal left ventricular mechanics of ventricular ectopic beats: insights into origin and coupling interval in premature ventricular contractioninduced cardiomyopathy. Circ Arrhythm Electrophysiol. (2015) 8:1194200. doi: 10.1161/CIRCEP.115.003047

27. Torrado J, Kowlgi GN, Ramirez RJ, Balderas-Villalobos J, Jovin D, Parker $\mathrm{C}$, et al. Eccentric hypertrophy in an animal model of mid- and long-term premature ventricular contraction-induced cardiomyopathy. Heart Rhythm O2. (2021) 2:80-8. doi: 10.1016/j.hroo.2020.12.021

28. Walters TE, Szilagyi J, Alhede C, Sievers R, Fang Q, Olgin J, et al Dyssynchrony and fibrosis persist after resolution of cardiomyopathy in a swine premature ventricular contraction model. JACC Clin Electrophysiol. (2020) 6:1367-76. doi: 10.1016/j.jacep.2020.06.020 
29. Modin D, Biering-Sørensen SR, Møgelvang R, Jensen JS, Biering-Sørensen T. Prognostic importance of left ventricular mechanical dyssynchrony in predicting cardiovascular death in the general population. Circ Cardiovasc Imaging. (2018) 11:e007528. doi: 10.1161/CIRCIMAGING.117.0 07528

30. Gupta A, Harrington M, Albert CM, Bajaj NS, Hainer J, Morgan V, et al. Myocardial scar but not ischemia is associated with defibrillator shocks and sudden cardiac death in stable patients with reduced left ventricular ejection fraction. JACC Clin Electrophysiol. (2018) 4:120010. doi: 10.1016/j.jacep.2018.06.002

31. Wang Y, Eltit JM, Kaszala K, Tan A, Jiang M, Zhang M, et al. Cellular mechanism of premature ventricular contraction-induced cardiomyopathy. Heart Rhythm. (2014) 11:2064-72. doi: 10.1016/j.hrthm.2014. 07.022

32. Hamon D, Rajendran PS, Chui RW, Ajijola OA, Irie T, Talebi R, et al. Premature ventricular contraction coupling interval variability destabilizes cardiac neuronal and electrophysiological control: insights from simultaneous cardioneural mapping. Circ Arrhythm Electrophysiol. (2017) 10:e04937. doi: 10.1161/CIRCEP.116.004937

33. Smith ML, Hamdan MH, Wasmund SL, Kneip CF, Joglar JA, Page RL. High-frequency ventricular ectopy can increase sympathetic neural activity in humans. Heart Rhythm. (2010) 7:497-503. doi: 10.1016/j.hrthm.2009. 12.029

34. Tan AY, Elharrif K, Cardona-Guarache R, Mankad P, Ayers O, Joslyn M, et al. Persistent Proarrhythmic Neural Remodeling Despite Recovery From Premature Ventricular Contraction-Induced Cardiomyopathy. J Am College Cardiol. (2020) 75:1-13. doi: 10.1016/j.jacc.2019.10.046

35. Voskoboinik A, Hadjis A, Alhede C, Im SI, Park H, Moss J, et al. Predictors of adverse outcome in patients with frequent premature ventricular complexes: the ABC-VT risk score. Heart Rhythm. (2020) 17:106674. doi: 10.1016/j.hrthm.2020.02.020

36. Yamada S, Yoshihisa A, Sato T, Kamioka M, Kaneshiro T, Oikawa M, et al. Prognostic significance of premature ventricular complex burden on hospitalized patients with heart failure. J Arrhythmia. (2020) 36:13442. doi: 10.1002/joa3.12259

37. Chen YS, Chen WD, Chen YH, Akhmerov A, Wang HC, Chang KC, et al. Atrial and ventricular response to treatment of premature ventricular complexes. Acta Cardiol Sin. (2020) 36:475-82. doi: 10.6515/ACS.202009_36(5).20200307A

Conflict of Interest: The authors declare that the research was conducted in the absence of any commercial or financial relationships that could be construed as a potential conflict of interest.

Publisher's Note: All claims expressed in this article are solely those of the authors and do not necessarily represent those of their affiliated organizations, or those of the publisher, the editors and the reviewers. Any product that may be evaluated in this article, or claim that may be made by its manufacturer, is not guaranteed or endorsed by the publisher.

Copyright (c) 2022 Lee, Huang, Huang, Hsu, Su, Liu, Hung and Liu. This is an open-access article distributed under the terms of the Creative Commons Attribution License (CC BY). The use, distribution or reproduction in other forums is permitted, provided the original author(s) and the copyright owner(s) are credited and that the original publication in this journal is cited, in accordance with accepted academic practice. No use, distribution or reproduction is permitted which does not comply with these terms. 